\title{
Characterization of flexure hinges for the French watt balance experiment
}

\author{
Patrick Pinot ${ }^{1 \mathrm{a}}$ and Gérard Genevès ${ }^{2}$ \\ ${ }^{1}$ Conservatoire National des Arts et Métiers (LCM) 61, rue du Landy 93210 La Plaine Saint-Denis, France \\ ${ }^{2}$ Laboratoire National de métrologie et d'Essais (LCM) 29, av. Roger Hennequin, 78197 Trappes Cedex, France
}

\begin{abstract}
In the French watt balance experiment, the translation and rotation functions must have no backlash, no friction, nor the need for lubricants. In addition errors in position and movement must be below $100 \mathrm{~nm}$. Flexure hinges can meet all of these criteria. Different materials, profile shapes and machining techniques have been studied. The flexure pivots have been characterized using three techniques: 1) an optical microscope and, if necessary, a SEM to observe the surface inhomogeneities; 2) a mass comparator to determine the bending stiffness of unloaded pivots; 3) a loaded beam oscillating freely under vacuum to study the dynamic behavior of loaded pivots.
\end{abstract}

\section{Introduction}

Since 2002 French metrology has been committed to a watt balance experiment [1], its aim is to determine Planck's constant with a relative uncertainty of order of $10^{-8}$ and thereby redefine the SI kilogram. In such an experiment, the weight of a mass standard is first balanced by the electromagnetic force exerted on a current carrying coil in a magnetic field. In a second dynamic phase, the coil is displaced at a known velocity and the induced emf measured. The combination of both phases allows one to eliminate the magnetic field and coil geometry. The static phase of all watt balances resembles that of the electrodynamometer previously used to materialize the definition of the SI ampère and thus the subject of considerable development.

The mechanical heart of the French watt balance is made up of a balance beam similar to that of a mass comparator. Given the final uncertainty expected, the repeatability of the balance must have a relative standard uncertainty less than $10^{-8}$ when forces of a few newtons are compared. This accuracy level is achieved in the present mass comparators by replacing, among other things, contact pivots (i.e. knife/edge) by flexure-strip pivots.

This type of flexure hinge is used in many high-accuracy measurement instruments (gyroscope, seismometer, etc) whose mechanical parts are limited in rotation $(<10$ mrad) or translation $(<100 \mu \mathrm{m})$. In the French watt balance experiment, flexure pivots are implemented for most hinges and guidance systems (vertical translation system, gimbals decoupling mass and coil suspensions, balance beam...).

This kind of pivot has the advantage of working by elastic deformation, implying no backlash, no friction and nor the need for lubricants. In addition they can be used under vacuum. To optimize the effectiveness of such pivots, one needs to quantify their mechanical properties of bending, twisting, translation, etc. This allows one to choose the best appropriate material, and the shape and dimensions of the flexible element that offer a wide elastic range, sufficient tensile strength and low thermoelastic energy dissipation. Of course, technical limitations such as the applicable machining methods depending on the material and the geometry of the profile must be also taken into account.

After a theoretical study [2] for designing a mechanical beam balance for the French experiment, we have undertaken several studies specifically on flexure pivots [3-5]. At present, supplementary studies are in progress in the scope of the Joint Research Project kNOW whose the main objective is to reduce the measurement uncertainty up to the $2 \times 10^{-8} \mathrm{~h}$ level required to redefine the kilogram.

After presenting briefly some appropriate materials, available machining techniques and characterization methods used, we provide some illustrative examples of results.

\section{Physical characteristics}

\subsection{General considerations}

In the French experiment, the moving end of the flexible pivots is mainly subjected to a system of tensions and shear forces that can reach tens of newtons (e.g. the central pivot of the balance beam must support a weight of about $60 \mathrm{~N}$ ). The angular strokes are of very low amplitude, generally less than $10 \mathrm{mrad}$. The first parameter to take into account is the uniaxial stress $W /(w \times e)$ where $W$ is the load, $w$ is the width of the flexible pivot and $e$ is the thickness of the thinner flexible part. This parameter must be smaller than the ultimate tensile strength of the material so as to avoid microscopic cracks and fracture.

Secondly, the design of a flexible hinge must take into account the largest movement in rotation or translation that it can support without failure or plastic deformation. When the flexure element is subject to pure bending, the stress experienced at the furthest points from the neutral axis of the beam must be smaller than the yield stress. Otherwise plastic deformations may appear on the extreme fibers where the compressive and tensile forces are the largest. Moreover, the plastic hinge state limit varies as a function of the number of stress cycles and depends on the limits of fatigue and endurance of the material depending themselves on the cycle frequency and load. In addition, there are different factors

a patrick.pinot@cnam.fr 
modifying these limits such as surface finish, size (scale effect), temperature, microstructure, residual stresses, shape discontinuities, corrosion, etc. In terms of material strength, damage is cumulative.

Thirdly, to reach the expected performance for our experiment, the flexure hinges must limit errors in position and movement below $100 \mathrm{~nm}$.

Amongst the physical parameters allowing one to characterize a flexure-strip, stiffness is one of the most important; it depends not only on the material, but also on the shape (size and profile) and moreover on how forces and moments are applied.

\subsection{Some types of flexure-strip}

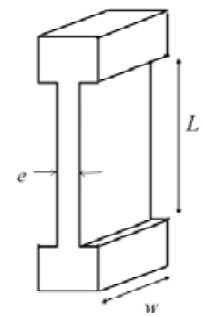

parallel faces

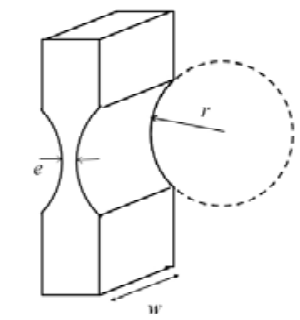

cylindrical neck

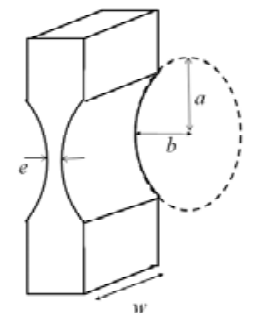

elliptical neck
Figure 1. Drawing of the three main types of flexure hinges

\subsubsection{Hinge with parallel faces}

The advantage offered by a flexible pivot with a constant rectangular section (i.e. with parallel faces) is that it is generally easy to make. It may be obtained either from a thick plate which is thinner at its central part by appropriate machining, or from a thin sheet which is clamped between two jaws at both ends. The main disadvantage of this kind of hinge is shape discontinuity. Moreover, the position of the rotation axis varies with the tensile load. Note that thin sheet down to $10 \mu \mathrm{m}$ thickness is commercially available allowing one to obtain a very weak stiffness, but it is very difficult to clamp the strips without stress (shear force, sliding...) and to adjust them correctly.

The bending stiffness $k_{1}$ of a flexible pivot with a constant rectangular section without tensile load is expressed as:

$$
k_{1}=\frac{E w e^{3}}{12 L}
$$

where $L$ is the length, $w$ the width of the flexible pivot, $e$ the thickness of the strip and $E$ the Young's modulus of the material.

\subsubsection{Hinge with circular neck}

For a circular neck hinge, the axis of rotation lies at the neck itself. Moreover, it has greater rigidities of torsion and translation than a simple flexure-strip with the same dimension $(e, w, L)$ and there is no shape discontinuity.

Henein [6] then Merken and Debongnie [7, 8] developed analytical expressions for hinges with circular profile of radius $r$ and thickness $e$ at the neck. The ratio $r / e$ must be greater than 5 to avoid any stress concentration at the neck, but not too large in order to keep the rotation axis at the neck. If one assumes the expression for the bending stiffness $k_{2}$ is linear, it is given by:

$$
k_{2} \approx \frac{2 E w}{9 \pi} \sqrt{\frac{e^{5}}{r}}
$$

Under particular assumptions, an expression for the bending stiffness $k_{3}$ obtained by applying a shearing force can be determined using energy conservation. When a shearing force $F$ is applied at a distance $l$ from the center of the neck, this leads to a displacement $f$ of the application point of force $F$, the flexion angle of the hinge (bending deflection) is $\theta \approx f / l$. The mechanical work of the force $F$ to produce the displacement $f$ is equal to the elastic energy stored in the flexure hinge. In this case, the stiffness is:

$$
k_{3} \approx \frac{2 E w}{9 \pi d^{2}} \sqrt{\frac{e^{5}}{r}}
$$

Note that the dimension of $k_{2}$ is that of a force multiplied by a length whereas that of $k_{3}$ is a force divided by a length. This is the consequence of the way the forces are applied.

\subsubsection{Hinge with elliptical neck}

The elliptical neck has virtually the same benefits as a circular neck but with a slightly lower angular stiffness for the same thickness at the neck. Consequently, this leads to a larger angular magnitude in the elastic range.

Following the same approach as Merken [8], the approximate analytical expression of the bending stiffness $k_{4}$ for a hinge with elliptical neck is:

$$
k_{4} \approx \frac{E w \sqrt{b e^{5}}}{18 a \pi}
$$

where $a$ and $b$ are the semi-major and semi-minor axes of the ellipse.

\section{Machining}

\subsection{General considerations}

The analytical expressions for stiffness show that the thickness $e$ of the hinge is the parameter that has the greatest influence on the bending stiffness. Given the cross-section area $(w \times e)$, the smaller the thickness $e$ of 
the pivot, the smaller its stiffness. The objective is to machine hinges such that $10 \mu \mathrm{m} \leq e \leq 50 \mu \mathrm{m}$.

The technique used for machining the flexible hinge must ensure a constant thickness over its entire width to within a few percent. In addition, the machining must not alter the physical characteristics of the material.

In particular, the machining must lead neither to any noticeable anisotropy to the flexure hinges, nor to any area likely to be a fracture initiation and must not be subject to any angular strain beyond the elastic limit of the material.

Different machining techniques were considered.

\subsection{Machining Techniques}

\subsubsection{Electrochemical Machining (ECM)}

Electrochemical Machining was rejected due to prohibitive tool production cost.

\subsubsection{Ion-beam Machining}

At first sight, the ion-beam machining seemed an interesting solution. However metals are often considered "indestructible" by this technique, so it is necessary to test for each material. In addition, the machining rate is extremely slow, of the order of $1 \mu \mathrm{m}$ per hour. This is why it could only be used as a method of surface finishing for "wearable" materials. Given these restrictions, this machining technique has not been implemented in the studies up to now.

\subsubsection{Ultrasonic Machining}

Ultrasonic machining is mainly used for crystalline materials, therefore with a high a priori hardness. So it seemed interesting for hard alloys such as Havar (a cobalt-based superalloy), or \#851 (a platinum-based alloy), or Vitreloy 1B (a zirconium-based amorphous alloy). But the tests at Femto-ST (Besançon) showed that this machining was efficient for these alloys only for a very limited depth (for instance $200 \mu \mathrm{m}$ for Vitreloy 1B). Beyond this limit, it is not the material which is machined but it is the sonotrode (the tool) which is worn.

\subsubsection{Electrical Discharge Machining (EDM)}

Wire-cut EDM is an electro-thermal production process in which a thin single-strand metal wire (usually brass) in conjunction with de-ionized water (used to conduct electricity) cuts through metal using heat provided by electrical sparks. EDM can be used for many metal alloys including copper-based alloys such as $\mathrm{CuBe} 2$. However wire-cut EDM applied to the zirconium-based alloy Vitreloy $1 \mathrm{~B}$ has not yielded satisfactory results. The main drawback of this technique is that it creates a deconstructed surface layer a few micrometers thick.

\subsubsection{Ultra-Precision diamond-tool Machining}

In Ultra-Precision diamond-tool Machining, the tool used is primarily selected according to the nature of material to manufacture. All the materials are not machinable with single-crystal diamond because of chemical reactions. Generally, the diamond cutting is applied only for machining various soft nonferrous metals, remarkably ductile. Machinable shapes are also limited depending on the type of machines available. Of the five alloys selected, only the copper ones ( $\mathrm{CuBe} 2$ and Chrysocale) were machined using this technique.

\subsubsection{Ultra high speed Machining (UHSM)}

In this case, UHSM is used with an inclined diamond milling cutter with one or two teeth at $40000 \mathrm{rpm}$. This kind of machining allows one to obtain an elliptical profile and can be applied to copper or aluminum alloys. There is almost no heating of the surface during the machining, because the heat is mainly removed by the cutting chip. This machining has been already used for hinges made out of aluminum alloy (7075) with a $1 \mathrm{~mm}$ thick neck, but not for $e<50 \mu \mathrm{m}$. The difficulty is to constrain the work-piece strongly without inducing strains.

\section{Examples of experimental results}

\subsection{Effects of the machining technique}

After machining a flexible pivot, its surface quality is examined around the flexure area by means of an optical microscope.

Figure 2 shows the groove left by a diamond tool on a pivot with a semi-circular neck $(r=8 \mathrm{~mm}$ and $e=50$ $\mu \mathrm{m})$, made from Chrysocale (UE3Z9).

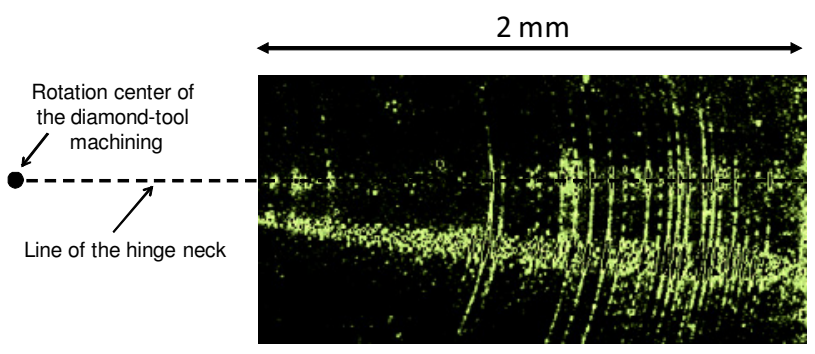

Figure 2. Photograph of the flexible area of a pivot with a semicircular neck $(r=8 \mathrm{~mm}$ and $e=50 \mu \mathrm{m})$, made from Chrysocale (UE3Z9) by Gaggione company by means of diamond-tool machining.

When necessary, a scanning electron microscope (SEM) can be used. For instance, observations with SEM of the flexible hinge, made from $\mathrm{CuBe} 2$, machined by wire-cut EDM at LNE allowed one to determine that the thickness of the disturbed metal surface layer was about $12 \mu \mathrm{m}$.

\subsection{Bending stiffness of a flexure pivot}




\subsubsection{Measurement method}

The technique for measuring the bending stiffness of a flexure pivot without tensile load consists in maintaining the pivot horizontally by one of its ends to a vertical positioner with a micrometer head. Its free end rests on a tip placed on the pan of a balance. The vertical translation $d$ of the fixed end of the pivot allows one to bend it more or less by a shearing force $F$ acting at the needle. This technique is similar to that used for the measurement of the very low bending force of an AFM tip [12].

Figure 3 shows a schematic diagram of the method used. In the experimental setup, the tip of $650 \mu \mathrm{m}$ radius is made from stainless steel. A laser sensor with a $10 \mathrm{~nm}$ resolution is used for measuring the vertical translation $d$. A balance with a $10 \mu \mathrm{g}$ resolution is used for determining the force $F$.

This quasi-static method can be used not only to determine the bending stiffness of a flexure pivot, but also its angular elasticity limit and any hysteresis phenomena.

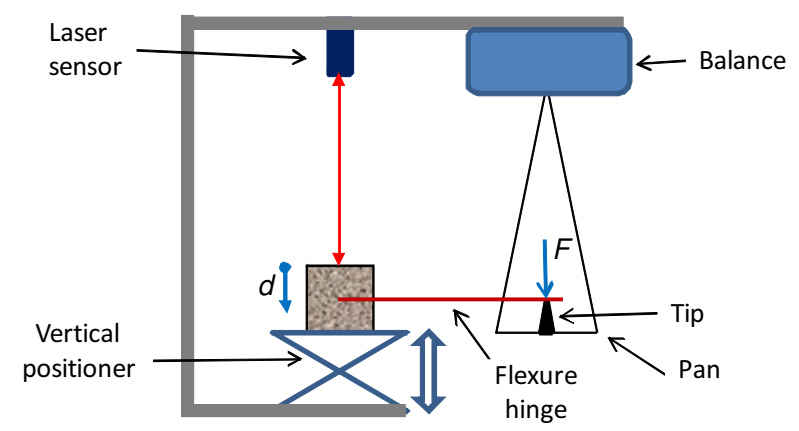

Figure 3. Diagram of the quasi-static method used for determining the bending stiffness of an unloaded flexure pivot.

\subsubsection{Examples of measurement results}

Figure 4 shows an example of increasing and decreasing bending deflection obtained under the same measurement conditions for two pivots with semi-circular neck $(r=8$ $\mathrm{mm}, e=50 \mu \mathrm{m}$ and $w=10 \mathrm{~mm})$, machined with diamond tool, one made from $\mathrm{CuBe} 2$ and the other from Chrysocale.

In this example, a displacement $\Delta d$ of $10 \mu \mathrm{m}$ is equivalent to a bending angle $\Delta \theta$ of $1.5 \mathrm{mrad}$. There is no significant hysteresis phenomenon. A quasi linear behavior is obtained over the explored range. This means that the deformation remains in the elastic range for both materials. An experimental stiffness coefficient $C$ can be determined as follows:

$$
C=g \frac{\Delta m}{\Delta d}
$$

where $g$ is the local acceleration due to gravity. It is assumed that horizontal force component is negligible regarding the very low values of bending deflection.

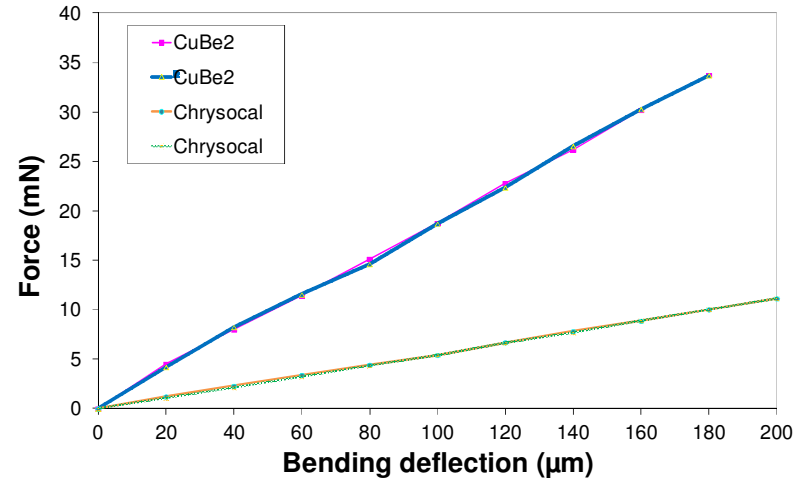

Figure 4. Curves of the restoring force of the pivot as a function of the increasing and decreasing bending deflection.

The ratio of the curve slopes (or coefficients $C$ ) between the two materials is 3.4 . Figure 4 shows that Chrysocale has a bending stiffness without tensile load lower than $\mathrm{CuBe} 2$. For the same geometry and dimensions, we can expect a better angular sensitivity and mobility of a balance beam with pivots made from Chrysocale rather than made from $\mathrm{CuBe} 2$.

In some cases, there may be a slight hysteresis phenomenon. For example, for pivots with a circular neck ( $r=3 \mathrm{~mm}, e=20 \mu \mathrm{m}, w=7.5 \mathrm{~mm}$ ), made from $\mathrm{CuBe} 2$ and machined by EDM, the mean slope of the experimental curves corresponded to a stiffness coefficient $C=18.5 \mathrm{~N} / \mathrm{m}$ according to expression (5). From these experimental results, we can also determine the coefficient $k_{3}$ from the analytical expression (3), which gives $k_{3}=37 \mathrm{~N} / \mathrm{m}$. The significant difference between the experimental and analytical values is partly explainable by the fact that the shearing force is applied differently. But it is likely that much of this difference is due to the metallographic structure inhomogeneity caused by EDM. In his book, Simon Henein [6] also admits that there is a neutral layer due to EDM which plays no role in the mechanical bending. Here, it is likely that wire-cut EDM changed the structure of the material over the thickness of the neck explaining the large difference between the $C$ and $k_{3}$ values.

\subsection{Thermo-elastic damping}

\subsubsection{Measurement method}

The dynamic behavior under vacuum of different flexible pivots is characterized in terms of thermo-elastic damping causing a hysteretic behavior of the pivot for different loads and oscillation frequencies. For this, a heavy oscillating beam was designed (see Figure 5).

It consists of:

- A beam made from aluminum alloy, of $520 \mathrm{~mm}$ long and $1.2 \mathrm{~kg}$;

- Two sliding cylindrical stainless steel masses which can be adjusted symmetrically to three different load values $(2.7 \mathrm{~kg}, 3.7 \mathrm{~kg}$ and $4.7 \mathrm{~kg}$ ); - Two threaded stems each having two knurled nuts to adjust the equilibrium of the beam; 
- Two permanent magnets at each beam end to drive the beam oscillation by means of a magnetic circuit; - A system of clamps at the center of the beam to secure the pivot to be studied.

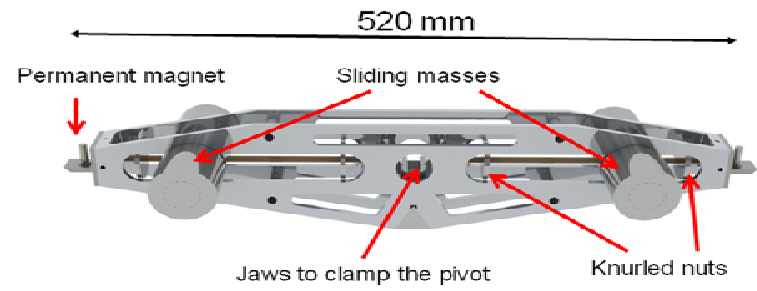

Figure 5. Drawing showing a perspective view of the heavy oscillating beam.

We measure the small rotations of the beam in vertical and horizontal planes corresponding to the bending and torsion of the hinge using optical detection, namely the movement of a laser beam reflected from mirrors glued to the end of the beam. Figure 6 presents a schematic diagram of the heavy oscillating beam. Only one of the optical detection systems and one ofthe magnet/coil electromagnetic motors are shown in Figure 6. The centre of rotation $\mathrm{O}$ of the flexure hinge must lie very close to the centre of mass of the oscillating beam.

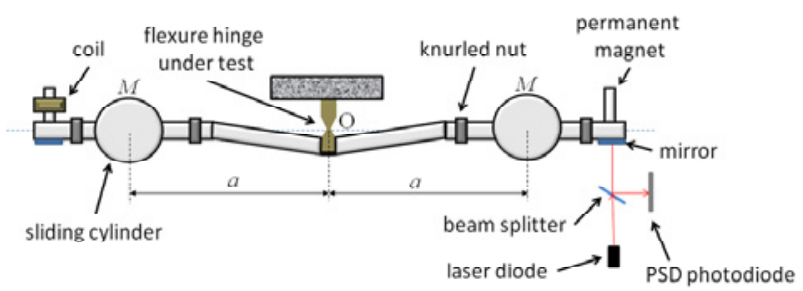

Figure 6. Schematic diagram of the heavy oscillating beam.

If the thermo-elastic damping of the material is essentially viscous, the angular magnitude of the damped oscillations can be expressed as follows:

$$
\theta(t)=\theta_{0}+\theta_{1} \sin (\omega t+\varphi) e^{-\frac{t}{\tau}}
$$

where $\theta(t)$ is the angular oscillation magnitude at time $t, \theta_{0}$ the angular magnitude shift for a mean value of $0, \theta_{1}$ the magnitude factor of the sinusoid, $\omega$ the pseudopulsation of the damped oscillation, $\varphi$ the phase, $t$ the time and $\tau$ the damping decay constant.

Based on equation (6), the damping decay constant $\tau$ and the pseudo-period $\omega$ were determined by numerical fitting using the curve fitting tool of MATLAB ${ }^{\circledR}$ software.

The well-known differential equation for oscillatory angular motion for this dissipative system can be written as:

$$
J \frac{d^{2} \theta}{d t^{2}}+B \frac{d \theta}{d t}+k_{5} \theta=0
$$

where $J$ is the moment of inertia of the heavy oscillating beam;

and $B$ is a damping coefficient which characterizes the thermo-elastic energy dissipated in the material of the flexure hinge such that:

$$
B=\frac{2 J}{\tau}
$$

where $k_{5}$ is the restoring moment coefficient. As for adjusting a beam balance [13], the centre of mass of the heavy oscillating beam is adjusted with respect to its centre of rotation in order to maximize the contribution of the hinge stiffness to the restoring moment. Thanks to this adjustment, the coefficient $k_{5}$ depends mainly on the bending stiffness under load of the flexure hinge. It can be determined from the following approximate expression:

$$
k_{5} \approx J\left(\omega^{2}+\frac{1}{\tau^{2}}\right)
$$

Integration of the equation (7) yields the energy equation:

$$
\begin{aligned}
\frac{1}{2} J\left(\frac{d \theta}{d t}\right)^{2}+\frac{1}{2} k_{5} \theta^{2}= & \frac{1}{2} M g a \theta_{\max }^{2} \cos ^{2}(\omega t+\phi)+ \\
& +\frac{1}{2} k_{5} \theta_{0}^{2}-\int_{0}^{t} B\left(\frac{d \theta}{d t}\right)^{2} d t
\end{aligned}
$$

where $M$ is the mass of each sliding cylinder and $a$ the distance between the centre of rotation of the heavy beam and the centre of mass of the cylinders.

The energy dissipated in the material is given by:

$$
E_{d}(t)=\int_{0}^{t} B\left(\frac{d \theta}{d t}\right)^{2} d t
$$

The expression for the $\log$ decrement $\Lambda$ is:

$$
\Lambda=\frac{1}{n} \ln \left(\frac{\theta_{0}}{\theta_{n}}\right)=\frac{2 \pi}{\omega \tau}=\frac{\pi B}{J \omega}
$$

\subsubsection{Examples of measurement results}

Table 1 presents the results for flexure pivots with a semi-circular neck $(r=8 \mathrm{~mm}, e=50 \mu \mathrm{m}$ and $w=10$ $\mathrm{mm}$ ). These pivots are made from $\mathrm{CuBe} 2$ and Chrysocale by ultra-precision diamond tool machining.

The moment of inertia $J$ is determined by numerical calculation using the COSMOS tool of SOLIDWORKS ${ }^{\circledR}$ software. 
The $\mathrm{CuBe} 2$ pivot under a $59 \mathrm{~N}$ load presents an unexpected behavior. Its stiffness is much lower than that under a $39 \mathrm{~N}$ load. It seems that the elastic properties of the material are modified due to a fatigue effect. The decrease of the damping coefficient $B$ for a $59 \mathrm{~N}$ load from $1.01 \mathrm{~mJ} \mathrm{~s}$ down to $0.68 \mathrm{~mJ} \mathrm{~s}$ may be explained by structural material damage. In contrast, the damping coefficient $B$ of the Chrysocale pivot remains relatively constant for different configurations with a mean value close to $0.7 \mathrm{~mJ}$ s.

\begin{tabular}{|c|c|c|c|c|c|c|c|}
\hline Material & $\begin{array}{c}\text { Mass } \\
\begin{array}{c}M \\
(\mathrm{~kg})\end{array} \\
\end{array}$ & $\begin{array}{c}\begin{array}{c}\text { Moment of } \\
\text { inertia }\end{array} \\
\underset{\left(\mathrm{kg} \cdot \mathrm{m}^{2}\right)}{ }\end{array}$ & $\begin{array}{c}\begin{array}{c}\text { Pseudo- } \\
\text { pulsation }\end{array} \\
\begin{array}{c}\omega \\
(\mathrm{rad} / \mathrm{s})\end{array} \\
\end{array}$ & $\begin{array}{c}\text { Damping } \\
\text { decay } \\
\text { constant } \\
\tau \\
(\mathrm{s}) \\
\end{array}$ & $\begin{array}{c}\text { Stiffness } \\
\\
k_{5} \\
\text { (N.m/rad) } \\
\end{array}$ & $\begin{array}{c}\begin{array}{c}\text { Logarithmic } \\
\text { decrement }\end{array} \\
\Lambda\end{array}$ & $\begin{array}{c}\begin{array}{c}\text { Damping } \\
\text { Coefficient }\end{array} \\
\boldsymbol{B} \\
(\mathrm{mJ} . \mathrm{s}) \\
\end{array}$ \\
\hline CuBe2 & 3.9 & 0.040 & 0.907 & 83 & 0.033 & 0.083 & 0.96 \\
\hline Chrysocale & 3.9 & 0.041 & 0.594 & 113 & 0.014 & 0.094 & 0.73 \\
\hline CuBe2 & 3.9 & 0.092 & 0.655 & 204 & 0.039 & 0.045 & 0.90 \\
\hline Chrysocale & 3.9 & 0.104 & 0.405 & 323 & 0.017 & 0.048 & 0.64 \\
\hline CuBe2 & 5.9 & 0.058 & 0.294 & 115 & 0.005 & 0.186 & 1.01 \\
\hline Chrysocale & 5.9 & 0.060 & 0.645 & 164 & 0.025 & 0.059 & 0.73 \\
\hline CuBe2 & 5.9 & 0.173 & 0.277 & 512 & 0,013 & 0.044 & 0.68 \\
\hline Chrysocale & 5.9 & 0.180 & 0.324 & 483 & 0,019 & 0.041 & 0.76 \\
\hline
\end{tabular}

Table 1. Examples of results obtained for pivots with a semicircular neck of $50 \mu \mathrm{m}$ thick

\section{Conclusions}

Three characterization methods are implemented for comparative testing on the flexure hinges used as pivots in the French watt balance experiment. In addition to the examination of the flexure pivot surface by optical microscope, two experimental methods were developed: a static one based on a weighing balance to compare the stiffness of unloaded pivots bended by a shearing force, and a dynamic one based on a freely oscillating heavy beam under vacuum to study the damping coefficient of pivots under different configurations in terms of stress frequency and load.

By some illustrative examples, this paper has shown how difficult it is to find the best machining technique suitable for the chosen material and the geometry of the flexure pivot. The characterization methods implemented allow one to select both the materials and the machining techniques by comparing the results obtained on pivots having the same geometry. The objective is to find the best compromise between the theoretical physical characteristics of a material, the machinable shape of the pivot and its static and dynamic behaviors in experimental conditions (weak stiffness, no hysteresis after several load cycles, etc).

For example, the study of pivots made from $\mathrm{CuBe} 2$ and Chrysocale, with the same geometry machined by diamond tool shows that Chrysocale (a copper-zinc-tin alloy) is a better candidate than $\mathrm{CuBe} 2$ (a copperberyllium alloy) for the balance beam of the French experiment. Given these results, although flexure pivots made from Chrysocale or from $\mathrm{CuBe} 2$ both present no hysteresis when they are submitted to static bending deflection, it seems that Chrysocale would provide a better sensitivity and mobility given its small stiffness coefficient for the watt balance beam and, therefore, a better repeatability of measurements. In addition, it seems that the elastic properties of $\mathrm{CuBe} 2$ are modified due to fatigue following repeated oscillation of the heavy beam.

For other materials such as Havar, \#851 or Vitreloy 1B, it remains to solve some difficulties in machining before studying their static and dynamic behaviors.

\section{"This work was funded through the European Metrology Research Programme (EMRP) Project SIBO3 $\mathrm{kNOW}$. The EMRP is jointly funded by the EMRP participating countries within EURAMET and the European Union."}

\section{References}

1. G. Genevès, P. Gournay, F. Villar, P. Pinot, P. Juncar, M. Lecollinet, L. Chassagne, A. Clairon, A. Landragin, D. Holleville, F. Pereira Dos Santos, J. David, M. Besbes, F. Alves, S. Topçu, D. Haddad, A. Gosset, Z. Silvestri, P.-A. Meury, T. Madec, S. Macé, Revue Française de Métrologie 9, 2 - volume 1 (2007)

2. P. Pinot, G. Genevès, D. Haddad, J. David, P. Juncar, M. Lecollinet, S. Macé and F. Villar, Rev. Sci. Instrum. 78, 095108 (2007).

3. P. Pinot, S. Macé, G. Genevès, P. Gournay, D. Haddad, M. Lecollinet, F. Villar, M. Himbert, Revue Française de Métrologie 21, 2010-1 (2010)

4. F. Villar, J. David, G. Genevès, P. Juncar and P. Pinot, "Long stroke flexure stage for a watt balance experiment", EUSPEN 6th International Conference, Baden, Autriche (2006).

5. P. Pinot, S. Macé, G. Genevès, P. Gournay, D. Haddad, M. Lecollinet, F. Villar and M. E. Himbert, Eur. Phys. J. Appl. Phys. 44, 2 (2008)

6. S. Henein, «Conception des guidages flexibles », Presses Polytechniques et Universitaires Romandes (2004)

7. P. Merken and J. F. Debongnie, «Le col circulaire comme articulation flexible , Proceeding of the $6^{\text {th }}$ National Congress on Theoretical and Applied Mechanics, Ghent, NCTAM-2003-077 (2003).

8. P. Merken, «La fonction guidage en micromécanique », thèse de DEA, université de Liège (2006)

9. T. J. Quinn, C. C. Speake and R. S. Davis, Metrologia $23(1986 / 87)$

10. T. J. Quinn, Meas. Sci. Technol. 3 (1992)

\section{A. Picard, Metrologia 41 (2004)}

12. M.-S. Kim, J.-H. Choi, Y.-K. Park and J.-H. Kim, Metrologia 43, 5 (2006)

13. C. C. Speake, Proc. R. Soc. London, Ser. A 414, 333 (1987). 